\title{
DISTÚRBIOS DE MEMÓRIA EM PACIENTES EPILÉPTICOS
}

\author{
FLORINDO STELLA
}

\begin{abstract}
RESUMO - O autor estudou distúrbios de memória em pacientes epilépticos com crises parciais complexas (CPC), com os objetivos de: 1) identificar estes distúrbios; e 2) comparar os resultados dos pacientes com os dos controles. Foram estudados 50 pacientes adultos e 20 sujeitos sem enfermidades neuropsiquiátricas. Os métodos consistiram em: 1) investigação da atividade mnemônica através do Teste de Memória de Wechsler (subtestes: Armazenamento e Recuperação, Memória Recente e Memória Imediata); e 2) comparação entre os resultados de ambos os grupos. 3) associação entre SPECT Cerebral e atividade mnemônica. Nos três subtestes, os pacientes apresentaram desempenho cognitivo significativamente inferior ao dos controles ( $\mathrm{p}<0,05)$. Constatou-se a associação entre hipofluxo em região temporal esquerda e distúrbio da memória nos três subtestes. Concluiu-se que há associação entre CPC e distúrbios da atividade mnemônica.
\end{abstract}

PALAVRAS-CHAVE: distúrbios de memória, avaliação neuropsicológica, epilepsia de lobo temporal.

\section{Memory disorders in epileptic patients}

ABSTRACT - The author studied the mnemonic activity from epileptic patients with complex partial seizures (CPS), with the aims: 1) to identify memory disorders; and 2) to compare the patients' with the controls' results. Fifty adult patients and 20 subjects without neuropsychiatric disorders were studied. The methods consisted in: 1) investigation of the mnemonic activity through the Wechsler Memory Test (subtests: Storage and Recall, Recent Memory, and Immediate Memory); 2) comparison among the results of both groups; association from mnemonic activity with brain SPECT. In the three subtests, the patients showed cognitive performance significantly lower than the controls $(p<0,05)$. It was found association from reduced blood flow, mainly in left temporal region, with memory impairment of the three subtetsts. The conclusion was that the CPS are associated to memory impairment.

KEY WORDS: memory disorders, neuropsychological evaluation, temporal lobe epilepsy.

A epilepsia de lobo temporal (ELT) tem sido associada a disfunções cognitivas, particularmente a distúrbios de memória de curta e de longa duração, identificáveis pela avaliação neuropsicológica ${ }^{1-3}$. Vários estudos têm documentado a presença de deficit de memória em pacientes epilépticos de difícil controle, principalmente quando o foco epileptogênico localiza-se em lobo temporal ${ }^{4-7}$. Delaney et al. ${ }^{4}$, estudando o processo mnemônico em pacientes portadores de epilepsia focal, encontraram distúrbios de memória, principalmente de longo prazo, em portadores de crises parciais complexas (CPC). Estas são crises com origem em área circunscrita do cérebro, geralmente de lobos temporais, em que há comprometimento da consciência. Elas podem ou não evoluir para generalização secundária. Delaney et al. ${ }^{4}$ constataram que, quando as crises tinham origem em lobo temporal esquerdo, a perturbação atingia o armazenamento de informações verbais; e quando o acometimento originava-se no lobo temporal direito, o distúrbio refletia-se na retenção de informações não-verbais e vísuo-espaciais.

Estudo realizado no Serviço de Neuroepilepsia, Faculdade de Ciências Médicas da Universidade Estadual de Campinas (UNICAMP), como parte de Tese de Doutorado em Neurociências, apresentada em junho de 1998. Apoio FAPESP. Aceite: 3-fevereiro-1999.

Dr. Florindo Stella - Instituto de Biociências - Universidade Estadual Paulista, Campus de Rio Claro - Caixa Postal 199 - 13.506-900 Rio Claro SP - Brasil. 
Binnie et al. ${ }^{8}$ lembram que a dificuldade para processar, armazenar e recuperar informações está associada, em parte, à extensão e ao local de possíveis comprometimentos das estruturas cerebrais, ao grau de disfunção fisiológica, à frequência e severidade das crises, à neurotoxicidade das drogas antiepilépticas e ao estágio de desenvolvimento cognitivo do sujeito quando do início das crises. Em geral, é difícil determinar-se a importância relativa de cada fator. Rausch \& Babb ${ }^{9}$ esclarecem que há correlação significativa entre diferentes índices de comprometimento da memória verbal e a presença de diferentes graus de lesão em neurônios hipocampais de lobo temporal esquerdo, e estudos recentes, indicando esclerose hipocampal em pacientes com ELT, têm sido vinculados ao comprometimento da atividade mnemônica ${ }^{10,11}$. Comparando os resultados de avaliação neuropsicológica de pacientes portadores de CPC recentemente diagnosticadas com os dados de sujeitos normais, Ronnberg et al. ${ }^{12}$ verificaram que os pacientes apresentavam inabilidade para tarefas complexas que envolvessem a atividade mnemônica associada à execução delas. Além disso, esses autores constataram demora maior na execução de tarefas simples que exigissem o acesso à memória de longa duração. Relacionando pacientes adultos portadores de epilepsia recentemente diagnosticada e apresentando crises recorrentes não tratadas, com sujeitos normais pertencentes ao grupo de controle, Kalviainem et al. ${ }^{13}$ encontraram distúrbios de atenção e de memória que corroboram os dados obtidos por Ronnberg e colaboradores.

Aikia et al. ${ }^{14}$ estudaram as atividades de aprendizagem e memória verbal em 56 pacientes adultos com CPC recentemente diagnosticadas e de etiologia desconhecida, utilizando subtestes de aprendizagem e de memória da Escala de Wechsler de Inteligência para Adultos. Os resultados indicam que $52 \%$ dos pacientes evidenciaram distúrbios de memória verbal, sobretudo para evocação, enquanto que, no grupo de controle, o índice situou-se em 15\%. Resultados semelhantes foram verificados por Moore \& Baker ${ }^{15}$ mediante, também, a aplicação da Escala de Memória, de Wechsler.

Os objetivos deste estudo foram: a) descrever os distúrbios de memória em pacientes epilépticos, portadores de CPC; b) verificar a existência de associação entre SPECT cerebral e memória nos pacientes epilépticos; c) comparar os resultados dos testes alcançados pelos pacientes com os resultados obtidos por sujeitos sem enfermidades neuropsiquiátricas.

\section{MÉTODO}

Foram estudados 50 pacientes de ambos os sexos, com idade entre 19 e 49 anos, portadores de epilepsia CPC, em tratamento no Serviço de Neuroepilepsia, da Faculdade de Ciências Médicas da Universidade Estadual de Campinas, (UNICAMP). Para efeito comparativo, também foram investigados 20 sujeitos com características sócioeconômico-culturais e profissionais semelhantes às dos pacientes, e isentos de enfermidades neuropsiquiátricas.

O desenvolvimento da pesquisa percorreu várias etapas:

a) Estabelecimento dos critérios de inclusão: pacientes epilépticos, com CPC em atividade, comportamento de adesão ao tratamento (caracterizada pelo uso regular da medicação antiepiléptica, seguimento das prescrições médicas tais como realização dos exames complementares quando solicitados, retorno regular ao Serviço). Estabelecimento dos critérios de exclusão: presença de enfermidades neuropsiquiátricas ou sintomas de doenças mentais, particularmente os transtornos depressivos, que pudessem comprometer o desempenho cognitivo.

b) Aplicação do Teste de Memória, de Wechsler, com os subtestes: Armazenamento e Recuperação, Memória Recente e Memória Imediata.

c) Comparação entre os resultados do SPECT cerebral - HMPAO Tc 99m Interictal, efetuado nos pacientes com indicação clínica, e os dados do Teste de Memória.

d) Comparação do desempenho cognitivo dos pacientes com o dos sujeitos-controles.

Cabe realçar que o Teste de Memória de Wechsler é um instrumento de avaliação da memória amplamente utilizado nos estudos das funções cognitivas, em vários centros internacionais e em nosso meio, como na Unidade de Neuropsicologia da UNICAMP.

\section{RESULTADOS}

A média de idade dos pacientes estudados foi de 32 anos e 5 meses, e a dos controles, 36 anos e 6 meses ( $p>0,05)$. A média de escolaridade dos pacientes situou-se em 7 anos e 6 meses, e a dos controles, 7 anos e 4 meses ( $p>0,05)$. 
A idade de início das crises epilépticas, em média, ocorreu aos 14 anos e 1 mês, e a cronicidade delas tinha, em média, a duração de 18 anos e 1 mês. O intervalo médio interictal correspondia a um episódio a cada 33,4 dias. Do ponto de vista clínico e eletrencefalográfico, todos os pacientes apresentavam crises parciais com origem em lobo temporal, sendo que, na maioria dos pacientes (92\%), as crises se generalizavam.

Os medicamentos mais utilizados pelos pacientes eram: carbamazepina (30 pacientes: $60 \%)$, difenil-hidantoína (6 pacientes: 12\%), fenobarbital (1 paciente: $2 \%$ ), oxcarbazepina (1 paciente: $2 \%$ ). Em 12 pacientes (24\%) estava sendo utilizada associação de vários medicamentos diferentes com a finalidade de substituição de agentes farmacológicos.

A análise estatística dos resultados teve como base a aplicação do teste não-paramétrico de Mann-Whitney ${ }^{16,17}$.

\section{a) Armazenamento e Recuperação (Wechsler)}

Este subteste permite avaliar a qualidade da informação resgatada pelo sujeito. A média alcançada, aqui, pelos pacientes foi de 5,5 pontos, enquanto que os controles obtiveram a média de 11,0 pontos $(\mathrm{p}=0,0001)$.

\section{b) Memória Recente (Wechsler)}

O presente subteste tem o propósito de avaliar a capacidade de memorização para fenômenos apresentados ao sujeito pouco tempo antes de ele ter que recuperar as informações. A média alcançada pelos pacientes em Memória Recente foi de 11,4 pontos, e a média obtida pelos controles atingiu a 14,5 pontos $(\mathrm{p}=0,0002)$.

\section{c) Memória Imediata (Wechsler)}

Este subteste constitui-se de conjuntos numéricos e avalia a qualidade da memória imediatamente após a apresentação do estímulo. Os pacientes alcançaram a média de 27,0 pontos, enquanto que os controles, a média de 38,0 pontos ( $\mathrm{p}=0,0005)$.

Nos três subtestes acima, houve diferença estatisticamente significativa $(\mathrm{p}<0,05)$ entre os resultados alcançados pelos pacientes e os obtidos pelos controles (Fig 1).

Dos 50 pacientes estudados, 36 (72\%) foram submetidos ao SPECT cerebral. Destes, em 26 pacientes $(72,2 \%$ de 36$)$ verificou-se hipoperfusão cerebral com predomínio em região temporal, em 17 pacientes $(63,4 \%$ dos 26 com SPECT alterado), sendo $9(34,6 \%)$ à esquerda e $5(19,2 \%)$ à direita. Em 3 pacientes $(11,5 \%)$ houve hipoperfusão nas regiões temporais bilateralmente. Nos demais

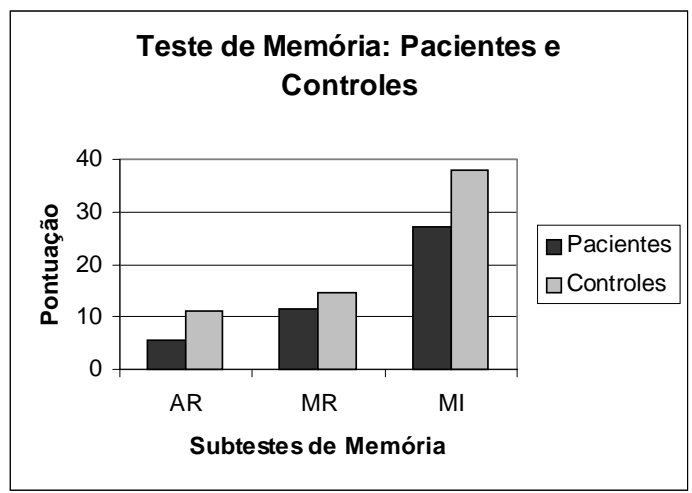

Fig 1. Gráfico evidenciando os resultados obtidos pelos pacientes (colunas mais escuras) e pelos controles (colunas mais claras) nos subtestes de Wechsler: AR (Armazenamento e Recuperação), MR (Memória Recente) e MI (Memória Imediata). 
9 pacientes 34,6\%), observou-se hipoperfusão difusamente. Os pacientes com hipofluxo em região temporal esquerda apresentaram desempenho cognitivo mais deficitário nos três subtestes (Armazenamento e Recuperação, Memória Recente e Memória Imediata) em comparação com os pacientes com hipofluxo em região temporal direita ou em outras áreas cerebrais.

\section{DISCUSSÃO}

Embora a literatura internacional mostre contingente elevado de pesquisas sobre as relações entre CPC e atividade mnemônica, em nosso meio os resultados de pesquisas sobre disfunções de memória em pacientes epilépticos adultos têm sido em número pequeno, e este dado levou o autor a investigar essas relações.

Os resultados mostram desempenho da memória significativamente inferior $(\mathrm{p}<0,05)$ entre os pacientes portadores de CPC, em comparação com os controles, nos três subtestes (Armazenamento e Recuperação, Memória Recente e Memória Imediata). Os dados sugerem que as CPC exercem algum tipo de comprometimento no processamento da memória dos pacientes. Um dos fatores que poderiam contribuir para a compreensão dos mecanismos responsáveis por esta interferência seria o fato de ambas - memória e CPC - serem processadas, pelo menos parcialmente, pela mesma área cerebral - o lobo temporal. Cabe lembrar que esta estrutura abrange o hipocampo, responsável pelo processamento da memória, e que, nos pacientes com $\mathrm{CPC}$, frequentemente se observa esclerose mesial temporal, por sua vez, uma das importantes causas das CPC.

$\mathrm{Na}$ clínica, frequentemente, a apresentação dos distúrbios de memória, pelo paciente com CPC, manifesta-se de maneira sutil. Entretanto, estes tornam-se evidentes através da aplicação de instrumentos específicos para a investigação da atividade mnemônica. Neste estudo, a utilização do Teste de Memória de Wechsler permitiu a identificação de diferença significativa $(\mathrm{p}<0,05)$ entre os resultados dos pacientes e dos controles. Assim, no subteste Armazenamento e Recuperação, os controles conseguiram o dobro dos pontos obtidos pelos pacientes. Nos subtestes Memória Imediata e Memória Recente, embora não se tenha verificado diferença tão acentuada, os pacientes também apresentaram desempenho significativamente inferior ao dos controles. Portanto, pela sua eficácia e facilidade de aplicação, o instrumento aqui utilizado para avaliação da memória constitui-se em recurso valioso ao clínico. Os resultados sugerem que se faça uso de instrumentos de avaliação da atividade mnemônica para a identificação de distúrbios de memória que, ao exame convencional, muitas vezes não são identificáveis.

Embora o estudo consista da avaliação de um processo psíquico específico - a memória - a atividade mental configura-se como uma totalidade indivisível. A partir de uma visão compreensiva do paciente epiléptico, admite-se que os processos psíquicos relacionam-se reciprocamente. Assim, a qualidade da memória, por um lado, depende particularmente da capacidade de concentração e de atenção. E, por outro, ela oferece elementos para o conteúdo do pensamento lógico. Vários estudos têm mostrado a interação destes processos psíquicos com a memória em pacientes com ELT $^{10,18-20}$. Os dados referentes ao desempenho cognitivo inferior dos pacientes no Teste de Memória, aqui utilizado, são concordantes, também, com os resultados de Kilpatrick et al ${ }^{21}$, que aplicaram subtestes da Escala de Wechsler de Inteligência para Adultos (WAIS-R) para a investigação da memória em pacientes epilépticos.

O desempenho cognitivo mais deficitário entre os pacientes com hipoperfusão em região temporal esquerda é concordante com pesquisas recentes que mostram deficit acentuado da atividade cognitiva em pacientes com ELT à esquerda. Os dados são compatíveis com resultados particularmente de investigações que mostram comprometimento de memória em pacientes com $\mathrm{CPC}^{1,7,22}$. A constatação de que pacientes epilépticos com hipoperfusão em região temporal, principalmente à esquerda apresentam desempenho cognitivo mais deficitário do que aqueles com hipoperfusão em outras áreas, reafirma os dados da literatura internacional. Além disso, a hipoperfusão cerebral em 
pacientes com CPC provavelmente represente um sinal de esclerose temporal mesial - alteração histopatológica compatível com a etiologia deste tipo de crise epiléptica. Embora tenha sido constatada hipoperfusão em outras áreas que não o lobo temporal, as CPC foram consideradas como tendo origem fundamentalmente em lobo temporal com base no perfil clínico das crises e nos exames eletrencefalográficos seriados, efetuados em cada paciente. Assim, permanece em aberto a necessidade de novas pesquisas que comparem a atividade cognitiva entre pacientes com CPC com origem em lobo temporal e aqueles com crises originárias de outras regiões cerebrais.

Para o tratamento clínico dos pacientes, além da necessidade de se considerar a condição epiléptica, é oportuno que se observe a qualidade das funções cognitivas, especificamente, o processo mnemônico. Um dos componentes desta proposta consiste na investigação das funções mnemônicas visando-se a manutenção e implementação delas. Treinamentos, na linha cognitiva, com a finalidade de se aprimorar a atividade mnemônica poderiam contribuir para sua preservação e aprimoramento.

Na avaliação neuropsicológica do paciente epiléptico caberia a questão: não haveria a presença de outros fatores envolvidos no desempenho cognitivo?

Certamente, há a interferência de vários componentes que, de forma direta ou indireta, podem influenciar o desempenho cognitivo dos pacientes, como tempo de duração e frequência das crises, medicação em uso, condições sócio-educacionais e atividade profissional. A dinâmica desses elementos torna extremamente complexa e, ao mesmo tempo, artificial, a tentativa de isolamento de qualquer uma dessas variáveis. A literatura admite que os medicamentos antiepilépticos, principalmente o fenobarbital, mas também a carbamazepina ${ }^{23-25}$, interferem nas funções cognitivas, com prejuízo das mesmas. Entretanto, vários autores afirmam que o controle das crises, em regime de monoterapia, com drogas mais recentes, provavelmente seja benéfico para as funções cognitivas ${ }^{26,27}$. No presente estudo, por questões operacionais, não foi possível identificar um grupo de pacientes que viessem ao tratamento sem medicação, uma vez que a grande maioria vinha encaminhada de outros serviços já em uso de agentes antiepilépticos.

A despeito da dificuldade de se isolar um dos componentes acima, é pertinente o esforço para se compreender as possíveis relações entre condição epiléptica e atividade mnemônica, particularmente em nosso meio.

\section{CONCLUSÃO}

a) Os pacientes apresentaram desempenho cognitivo significativamente inferior aos controles nos subtestes Armazenamento e Recuperação, Memória Recente e Memória Imediata.

b) $\mathrm{O}$ fato de as CPC, de modo geral, terem origem mesma área cerebral que processa a memória - o hipocampo - provavelmente contribua para a compreensão das relações entre CPC e distúrbios de memória. Pesquisas, em nosso meio, sobre esta questão, ainda deverão ser efetuadas. Além disso, não se pode omitir a interferência da medicação antiepilética nas funções cognitivas, particularmente, na memória.

c) Pacientes com hipofluxo em região temporal esquerda apresentaram desempenho cognitivo mais deficitário nos três subtestes (Armazenamento e Recuperação, Memória Recente e Memória Imediata) em comparação com os pacientes com hipofluxo em região temporal direita ou em outras áreas cerebrais.

d) Torna-se oportuna a inclusão da avaliação neuropsicológica na abordagem clínica do paciente epiléptico. Os subtestes de memória utilizados representam recursos úteis a serem incorporados aos procedimentos que visam investigar as repercussões que a condição epiléptica imprime na atividade cognitiva. Eles propiciam, ainda, subsídios clínicos para os programas de preservação da qualidade da memória. 


\section{REFERÊNCIAS}

1. Helmstaedter C, Gleissner R U, Di-Perna M, Elger CE. Relational verbal memory processing in patients with temporal lobe epilepsy. Cortex 1997;33:667-678.

2. Hershey T, Craft S, Glauser TA, Hale S. Short-term and long-term memory in early temporal lobe dysfunction. Neuropsychology 1998;12:52-64.

3. Jones-Gotman M, Smith ML, Zatorre RJ. Neuropsychological testing for localizing and lateralizing the epileptogenic region. In Engel J Jr. (ed). Surgical treatment of the epilepsies; Ed2. New York: Raven Press 1993;245-261.

4. Delaney RC, Rosen AJ, Mattson RH, Novelly RA. Memory function in focal epilepsy: a comparison of non-surgical, unilateral temporal lobe and frontal lobe samples. Cortex 1980;16:103-117.

5. Hermann BP, Wyler AR, Richey ET, Rea JM. Memory function and verbal learning ability in patients with complex partial seizures of temporal lobe origin. Epilepsia 1987;28,547-554.

6. Mungas D, Ehlens C, Walton N, McCuthen CB. Verbal learning differences in epileptic patients with left and right temporal lobe foci. Epilepsia 1985;26:340-345.

7. Pedley T. Neurobiologia da epilepsia de lobo temporal. In Guerreiro CAM, Guerreiro MM (eds).Epilepsia: 2Ed. S. Paulo: Lemos Editorial. 1996;19-29.

8. Binnie CD, Channon S, Marston D. Learning disabilities in epilepsy: neurophysiological aspects. Epilepsia 1990;31(Suppl 4):S2-S8.

9. Rausch R, Babb TL. Hipocampal neuron loss and memory scores before and after temporal lobe surgery for epilepsy. Arch Neurol 1993;50:812-817.

10. Baxendale AS, Van-Paesschen W, Thompson PJ, et al. The relationship between quantitative MRI and neuropsychological functioning in temporal lobe epilepsy. Epilepsia 1998;39:158-166.

11. Detre JA, Maccotta L, King D. et al. Functional MRI lateralization of memory in temporal lobe epilepsy. Neurology 1998;50:926-932.

12. Ronnberg J, Samuelsson S, Soderfeldt TB. Memory effects following carbamazepine monotherapy in patients with complex partial epilepsy. Seizure 1992;1:247-253.

13. Kalviainen PJ, Aikia M, Helkala EL, Mervaala E, Rickkinen PJ. Memory and attention in newly diagnosed epileptic seizure disorder. Seizure 1992;1:255-262.

14. Aikia M, Kalviainen R, Riekkinen P. Verbal learning and memory in newly diagnosed partial epilepsy. Epilep Res 1995;22:157-164.

15. Moore PM, Baker GA. Psychometric properties and factor structure of the Wechsler Memory Scale-Revised in a sample of persons with intractable epilepsy. J Clin Exper Neuropsychol 1997;18:897-905.

16. Agresti A, Finlay B. Statistical methods for the social sciences. 2Ed. San Francisco: Dellen Publ Co, 1986.

17. Conover WJ. Practical nonparametric statistics. New York: John Wiley \& Sons, 1971.

18. Aldenkamp AP. Effect of seizures and epileptiform discharges on cognitive function. Epilepsia 1997;38,(Suppl 1):S52-S55.

19. Burgess AP, Gruzelier JH. Localization of word and face recognition memory using topographical EEG. Psychophysiology 1997;34:7-16.

20. Mataro-Serrat M, Junqueira-Plaja C. Memory and epilepsy. Revista Neurológica (Barcelona)1997;25:1241-1245.

21. Kilpatrick C, Murrie V, Cook M, Andrewes D, Desmond P, Hopper J. Degree of left hippocampal atrophy correlates with severity of neuropsychological deficits. Seizure 1997;6:213-218.

22. Hermann BP, Seidenberg M, Schoendfeld J, Davies K. Neuropsychological characteristics of the syndrome of mesial temporal lobe epilepsy. Arch Neurol 1997;54:369-376.

23. Sabers A, Moller A, Dam M, Smed, A et al. Cognitive function and anticonvulsivant therapy: effects of monotherapy in epilepsy. Acta Neurol Scand 1995;92:19-27.

24. Hermann BP, Whitman S, Anton M. A multietiological model of psychological and social dysfunction in epilepsy. In Bennet T (ed). The neuropsychology of epilepsy. New York: Plenum Press, 1992;39- 57.

25. Bennett LT.Cognitive effects of epilepsy and anticonvulsivant medications. In Bennett LT (ed.). The neuropsychology of epilepsy. New York: Plenum Press, 1992;73-95.

26. Vermeulen J, Aldenkamp AP. Cognitive side-effects of chronic antiepileptic drug treatment: a review of 25 years of research. Epilep Res 1995;22:65-95.

27. Powell AL, Yudd A, Zee P, Mandelbaum DE. Attention deficit hyperactivity disorder associated with orbitofrontal epilepsy in a father and a son. Neuropsychiatry Neuropsychol Behav Neurol 1997;10:151-154. 